\title{
ROBOTICS IN ONLINE INSPECTION AND QUALITY CONTROL USING MOMENT ALGORITHM
}

\author{
Dev Anand, M.*; Selveraj, T. ** Kumanan, S. ${ }^{* *} \&$ Ajith Bosco Raj, T. ** \\ ${ }^{*}$ Corresponding Author, Professor, Department of Mechanical Engineering, Noorul Islam \\ University - 629 180a \\ **Department of Production Engineering, National Institute of Technology, Tiruchy -620 015 \\ ${ }^{* * *}$ Lecturer, Lord Jagannath College of Engineering and Technology, Tamilnadu, India \\ E-mail: anandpmt@yahoo.co.in
}

\begin{abstract}
:
In the past few years, robotics has been used in a wide range of industrial applications. Material transfer is one of the basic applications require the robot to move materials or work parts from one location to another. The important function involved in this area is typical inspection and quality control of job, the positions a sensor with respect to the work part moment and determines whether the part is consistent with the quality specifications. One of the benefits often claimed for robotics is that the quality of the manufactured product will improve when a robot performs the operation. This section examines the claim and suggests the possible sources of quality improvement that might result from the application of the robot as an alternative for human operator. Inspection is another area of factory operations in which there is a significant interest in automation. The potential has increased with the development of new sensors, vision processing equipments, micro-controllers and computers with better performance at lower cost. On-Line inspection using robots detects products out of tolerance at an early stage and reduces the need for rework as well as scrap. The systems are used to perform tasks which include selecting parts that are randomly oriented from a bin or conveyer, parts identification, and complex inspection for close dimensional tolerances, and improved recognition and part location capabilities. These will result from the constantly reducing cost of computational capability, increased speed, and new as well as better algorithms are currently being developed. Information about deviations from goal point may also be fed back to the process. In this paper we have used Moment Algorithm (MA) for robot online inspection and quality control.
\end{abstract}

Key Words: Industrial Robots, Inspection, CCD Cameras, Moment Algorithms

\section{INTRODUCTION}

System for automated inspection and quality control are becoming more widespread, due to a combination of developments made in the field of sensors and the desires to insure consistency of product quality. Product quality constitutes an important factor in modern manufacturing systems and unmanned or partially manned manufacturing systems, vision inspection systems, robot manipulated inspection, demands automated measuring and inspection systems in order to ensure product quality. Traditionally, inspection and quality control are performed manually, by the use of fixtures or coordinate measuring devices. However, in automated manufacturing systems, the absence of a human operator may invalidate these techniques. In order to detect deviations from the nominal values or to detect the absence of vital parts or properties, the inspection process should be performed on-line. The main benefit with on-line inspection is that products out of tolerance are detected at an early stage in the manufacturing process. This means that products out of tolerance are prevented from further machining, handling, assembly, etc., resulting in less rework and 
scrap. In addition, information on deviations from target values may be fed back to the process. The flexibility of robots in combination with more accurate, high performance sensors will provide solutions to different inspection tasks. Research on the utilization of moments for object characterization in invariant tasks has received considerable attention in recent years. The principal techniques explored include moment invariants, geometric moments, rotational moments, orthogonal moments, and complex moments, various forms of moment descriptors have been extensively employed as pattern features in scene recognition, registration, identification, object matching as well as data compression.

The uniqueness theorem states that if $f(\mathrm{x}, \mathrm{y})$ is piecewise continuous and has non zero values only in the finite part of the $(x, y)$ plane, then geometric moments of all orders exist. Since an image segment has finite area and, in the worst case, is piecewise continuous, a moment set can be computed and used to uniquely describe the information contained in the image segment. Using nonlinear combinations of geometric moments, a set of invariant moments which has the describe properties of being invariant under image translation, scaling, and rotation. Howe, the reconstruction of the image from these moments is deemed to be quite difficult. The rotational moment is an alternative to the regular geometric moment. The rotational moments are based on a polar coordinate representation of the image and can be used to extend the definition of moment invariants to arbitrary order in a manner which ensures that their magnitudes do not diminish significantly with increasing order. Complex moments are simple and straight forward way to derive a set of invariant moments, but not good image features. The orthogonal moments to recover the image from moments based on the theory of orthogonal polynomials. In general, orthogonal moments are better than other types of moments in terms of information redundancy and image representation. The mathematical concept of moments has been around for several years and has been used in many diverse fields ranging from mechanics, statistics to pattern recognition and image understanding. Describing images with moments instead of other more commonly used image features means that global properties of the image are used rather than local properties.

\section{DESIGN OF A ROBOTIZED INSPECTION SYSTEM}

Designing a flexible robotized system for inspection purposes requires several factors to be considered and selections to be made [1]. These can be summarized in the model shown in Figure1. The basic consideration is the task of the system. The task may be divided into two categories; inspection and quality control. Inspection deals with the objective to detect products out of tolerance or products missing vital properties such as, holes, parts, etc. This usually results in a Go/No Go decision. Quality control deals with questions such as how close is the product to the nominal target value. Once the task product of the system is defined the actual components required may be selected. The selection of a suitable robot constitutes an important factor since the performance of a system is often determined by the ability of the robot to position the end-effector correctly. Other aspects are work envelope, load carrying capacity and number of axes. The number of axes is important in terms of accessibility and the ability in orientating sensors. Based on the task, several suitable sensors may usually be considered that meet the required specifications in terms of accuracy, measuring range, disturbances, etc. Suitable sensor configurations include machine vision, laser sensors, ultrasonic sensors, range and proximity sensors. 


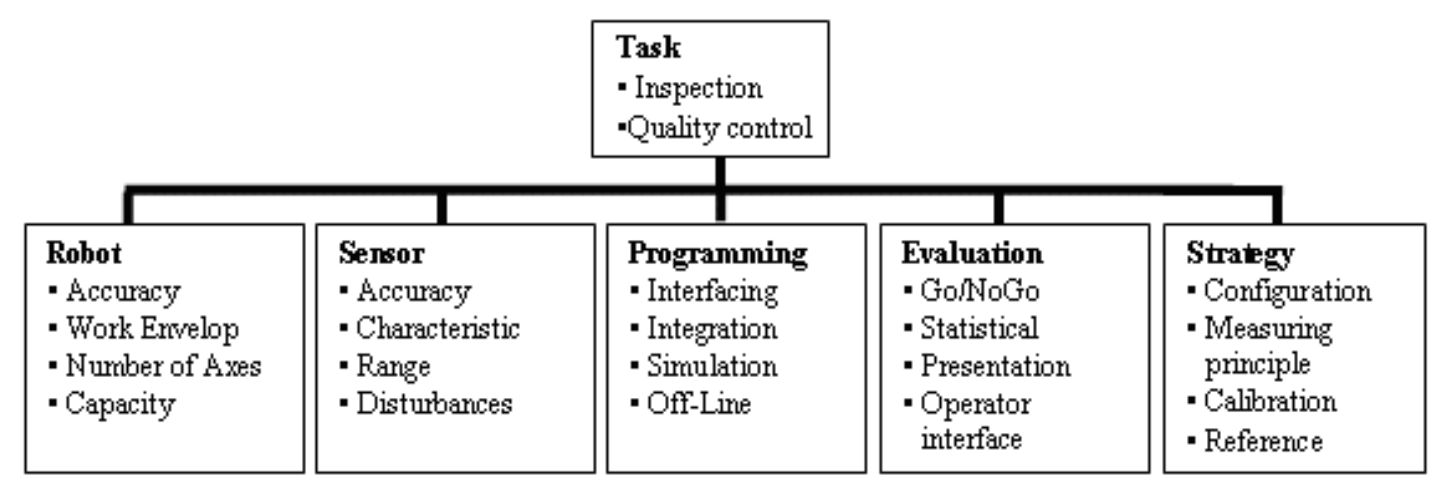

Figure1: Model for selection of components in a robotized system for inspection.

The programming of the system includes, apart from the actual programming of the robot, the design of the software with help of moment algorithm interfaces between the different systems. Regardless of the task, the signal from the different sensors must be analyzed and evaluated. This may include signal or image processing as well as improving the quality of the measured signal. The evaluation may result in a decision of whether the product may be passed on to the next stage in the manufacturing process, or error signals referring to how close to the target value based on the properties of the product is. The measuring strategy deals with the objective of combining all components in a system that is capable of performing all tasks required. When a robot is used, two basic configurations can be identified; robots configured with grippers and robots configured with sensors. The first approach is to let the robot present the work piece with separate unit to asset configuration of sensors and the second approach is the robot equipped with a configuration of sensors.

\section{ROBOT SELECTION}

The selection of a robot is very important since the overall performance of the system usually depends on the performance of the robot. The selection is influenced by several different factors that must be considered in detail but the most important factor is the performance of the robot. The performance usually focuses on the repeatability but the positioning accuracy and spatial resolution might also be of interest. There are several factors affecting robot performance and they may be classified in to the following categories [2].

- Environmental, such as temperature, humidity, structured, unstructured, hazards and domestic.

- Parametric, such as line length, structural compliance, back lash, slippage of pulley systems, deflection of manipulator links and other imperfections inherent in mechanical system.

- Application, such as installation, capability and fixture errors, operation is simple and repetitive, cycle time for the operation is very low, parts can be delivered to the operation in proper location and orientation, part weight suitable, setups and changes over are infrequent and fixture errors.

- Technical features, such as number of axes, type of control system, work volume, ease of programming, precision of motion, load handling capacity and multi pass features. Computational, such as errors in path, trajectory, task, grasp, motion, etc., and caused by computer round-off.

- Measurement, such as resolution and non-linearity in resolvers. 
Consider the most important environment factor is the temperature. During repetitive moments from a cold start, the influence of friction in the drive and the heat generation in motors causes possible change the link length. By measuring the temperature of the robot's structure a calibration function can be calculated which makes it possible to correct data obtained from different sensors.

\section{SENSOR SELECTION}

Various sensors can be used in robotic systems to achieve the good autonomous navigation. A large number of non contacting sensors suited for inspection and quality control purpose are commercially available, such as CCD, LED, laser and ultra sonic displacement sensors, profile scanners, vision system, proximity and range sensors. The basic consideration when selecting a suitable sensor is what properties of an object that is to be inspected or measured. Several factors affect the selection of sensor can be used in robotic system, such as:

- What resolution and accuracy, both absolute and repeatability is required?

- What kind of material does the object consist of?

- In what kind of environment will the sensor operate?

- What kind of communication interface is available?

- Geometrical constrains.

The type of environment which might be encountered, and its representation for planning should direct the choice of sensors in robotic systems. CCD cameras are the most obvious electronic parallel and computer vision system. However, for more general systems, particularly those in which there is cluster, there are some significant problems which remain to be solved especially in the image processing.

- Picking out the important information from very detailed images, especially in clustered environments or in varying the light conditions.

- Abstracting range information from intensity images.

- Achieving a high enough throughput rate.

Although vision systems are still expensive in compare with the simpler direct range sensors. CCD cameras measure intensity; for planning robots need to know range, stereo range measurement uses the difference in offset of features in the image between the two view points of components. The image processing acquires images from a miniature CCD camera situated by in front of the robot end-effectors. Edges are extracted using a dual convolution make and intelligent thresholding [3]. The edges are then thinned using morphological techniques [4]. The thinned edges in the image are the traced by the fitting straight lines to them, and turn into line vectors, which are then categorized in the basis of moment algorithm, range it estimated from the robot tool center point to camera geometry by assuming a flat earth. Inspection tasks, i.e. confirmation of certain properties such as presence of holes and those parts are assembled correctly usually requires different sensor characteristics in terms of accuracy than in the case of quality control.

\section{PROGRAMMING}

Programming includes robot programming, sensor integration and the communication interfaces between the robot controller and a supervisory host machine. The sensor interface is usually quite straight forward since the data collected by charge couple device through a standard communication interface. The interface to the robot controller usually constitutes a 
problem since no general purpose interface available. The communication interface to the robot is then reduced to simple digit input and output signals.

The programming of the robot is done by manually teaching a sequence of different positions and specifying velocities, type of motion, type of environment, etc. However, for the off-line programming the systems combined with flexible capabilities and stimulate a robot inspection program.

\section{EVALUATION}

The information and data obtained from different sensor systems must be analyzed and evaluated. This may include signal processing in order to improve the quality of the signal, by removing errors and noise as well as, for instance, edge detection, area calculation, etc. Based on tolerances and or target values specified for each parameter, actions may be taken. The objective of the inspection process might be to verify that the geometrical shape of the object is the object is within the control limits and or that certain attributes are correct. The result of this evaluation determines whether the product may be passed on to the next stage in the manufacturing process, if errors are detected the product is removed and the error is reported. In addition statistics may be compiled of the errors that have occurred.

\section{MEASURING STRATEGIES}

The Measuring strategy deals with the objective of combining all components in a system that is capable of performing all tasks required. The selection of a strategy is not an isolated part in the design of the system, it is affected by the selection of sensors, robot configuration and communication interfaces. However, there is one basic consideration, that is, whether the robot should be configured with a set of sensors or with a gripper. A robot configured with a gripper presents the object to a configuration of sensors. The robot is, in this case, regarded as a more or less simple device that handles different objects and presents them to a sensor system. This approach has the advantage that the inaccuracy of the robot in many cases can be disregarded; the performance of the system is the determined by the performance of the sensors. The second approach is to equip the robot's end-effector with a configuration of sensors; in this case the flexibility of the system may be increased. In order to achieve the highest accuracy possible, the performance of the robot must be optimized. The ability of the robot to position the end-effector at the desired position and orientation becomes the most important factor affecting the overall accuracy of the system. Three different strategies may be identified:

1. Positioning based on the repeatability of the robot.

2. Positioning based on the absolute accuracy of the robot.

3. Positioning based on information from external sensor.

In system based on the repeatability of the robot the end-effector of the robot is positioned in discrete location where the sensor is activated. By using a master work piece with known dimensions and/or attributes, the reading of the sensor may be compared with the performance on the master piece. For instance, if a high accuracy displacement sensor is used and the tool center point is well defined, the accuracy of the system will be very close to the repeatability of the robot. The objective of the inspection is to verify that the different surface lie with in the specified tolerances. In systems based on the absolute accuracy of the robot, the positioning information is obtained from the robot controller or the assumption that the desired position has been reached, where the robot has been moved in a preprogrammed pattern. By using a one-dimensional displacement sensor, it is possible to obtain a digitized image of the object. The different positions are specified relative to a 
starting position and if this position is known, the position of every other point in the pattern is known. Finally the position of the robot may be monitored by an external sensor system. This approach allows continuous measurements of the robot position and this means that the in accuracy of the robot can be disregarded and the overall accuracy is limited to the accuracy of the sensor and the external system. However, this approach is clearly limited by visibility and economical justification.

\section{METHODOLOGY OF WORK}

\subsection{Calculation of Moments}

\subsubsection{Calculation of Geometric Moment}

The two dimensional geometric moment of $\operatorname{order}(p, q)$ of a function $f(x, y)$ is defined as

$$
M_{p q}=\int_{-\infty-\infty}^{\infty} \int_{-\infty}^{\infty} x^{p} y^{q} f(x, y) d x d y
$$

Where $p, q=0,1,2 \ldots \infty$ Note that the monomial product $X^{p} Y^{q}$ is the basis function for this moment definition. The above expression can be implemented as follows

$$
M p q=\sum_{x=1}^{N 1} \sum_{y=1}^{N 2} x^{p} y^{q} f(x, y)
$$

Where

$\mathrm{N} 1=$ Total number of Pixels in horizontal axis

N2=Total number of Pixels in vertical axis

e.g.:-Consider an image of height and width are 3

$M[0][0]=1^{0} 1^{0 *}(\mathrm{a}[1][1])+1^{0} 2^{0 *}(\mathrm{a}[1][2])+1^{0} 3^{0 *}(\mathrm{a}[1][3])+2^{0} 1^{0 *}(\mathrm{a}[2][1])+2^{0} 2^{0 *}(\mathrm{a}[2][2])+2^{0} 3^{0 *}(\mathrm{a}[2][3])$ $+3^{0} 1^{0 *}(a[3][1])+3^{0} 2^{0 *}(a[3][2])+3^{0} 3^{0 *}(a[3][3])$

Where, $a[i][j]=$ pixel values. $i, j=1,2,3$.

\subsubsection{Calculation of Central Moments}

The central moments of $f(x, y)$ are defined as

$$
\mu_{p q}=\int_{a 1}^{a 2 b 2} \int_{b 1} X^{p} Y^{q} f(x, y)
$$

This can be expressed as follows

$$
\mu_{p q}=\sum_{x=1}^{N 1} \sum_{y=1}^{N 2}(X-\bar{X})^{p}(Y-\bar{Y})^{q} f(x, y)
$$

Where $\stackrel{\bar{X}}{=}=\mathrm{M}_{10} / \mathrm{M}_{00} \quad \bar{Y}=\mathrm{M}_{10} / \mathrm{M}_{00}$

$\mathrm{N} 1=$ Total number of Pixels in horizontal axis

N2=Total number of Pixels in vertical axis

The above expression can be implemented as follows

$\mu[0][0]=M[0][0] ;$ 
$\mu[0][1]=0 ;$

$\mu[0][2]=M[0][2]-\mathrm{y}^{*} M[0][1] ;$

$\mu[1][0]=0$;

$\mu[1][1]=M[1][1]-\mathrm{y}^{*} M[1][0] ;$

$\mu[1][2]=M[1][2]-2^{*} \mathrm{y}^{*} M[1][1]-\mathrm{x}^{*} M[0][2]+2^{*} \mathrm{y}^{*} \mathrm{y}^{*} M[1][0] ;$

$\mu[2][0]=M[2][0]-x^{*} M[1][0] ;$

$\mu[2][1]=M[2][1]-2^{*} \mathrm{x}^{*} M[1][1]-\mathrm{y}^{*} M[2][0]+2^{*} \mathrm{x}^{*} \mathrm{x}^{*} M[0][1]$;

$\mu[0][3]=M[0][3]-3^{*} \mathrm{y}^{*} M[0][2]+2^{*} \mathrm{y}^{*} \mathrm{y}^{*} M$ [0] [1];

$\mu[3][0]=M[3][0]-3^{*} \mathrm{x}^{*} M[2][0]+2^{*} \mathrm{x}^{*} \mathrm{x}^{*} M[1][0]$;

\subsubsection{Calculation of Normalized Central Moments}

The Normalized Central moments of $f(x, y)$ are defined as

$$
\eta_{p q}=\mu_{p q} / \mu_{p q}^{\gamma}
$$

Where $\gamma=(p+q) / 2+1$

$\eta[0][2]=\mu[0]$ [2]/pow $(\mu[0][0], 2)$;

$\eta[0][3]=\mu[0][3] / \operatorname{pow}(\mu[0][0], 2.5)$;

$\eta[1][1]=\mu[1][1] / \operatorname{pow}(\mu[0][0], 2)$;

$\eta[1][2]=\mu[1]$ [2]/pow ( $\mu$ [0] [0], 2.5);

$\eta[2][0]=\mu 2][0] /$ pow $(\mu[0][0], 2)$;

$\eta$ [2][1]= $\mu$ [2] [1] / pow ( $\mu$ [0] [0], 2.5);

$\eta[3][0]=\mu[3][0] /$ pow $(\mu[0][0], 2.5)$;

\subsubsection{Calculation of Seven Set of Invariant Moments}

The set of absolute moment invariants consists of a set of nonlinear combinations of central moments that remain invariant under rotation. Hu defines the following seven functions, computed from central moments through order three, that are invariant with respect to object scale, translation and rotation

$$
\begin{aligned}
& \varnothing[1]=n[2][0]+n[0][2 \\
& \varnothing[2]=(n[2][0]-\eta[0][2])^{*}(n[2][0]-\eta[0][2])+4^{*} \eta[1][1]{ }^{*} \eta[1] \\
& \varnothing[3]=\left(n[3][0]-3^{*} n[1][2]\right)^{*}\left(n[3][0]-3^{*} n[1][2]\right) \\
& +\left(3^{*} \eta[2][1]-\eta[0][3]\right)^{*}\left(3^{*} \eta[2][1]-\eta[0][3]\right) \text {; } \\
& \varnothing[4]=(n[3][0]-n[1][2])^{*}(n[3][0]-\eta[1][2])+(n[2][1]-\eta[0][3])^{*}(n[2][1]-\eta[0][3]) ; \\
& \varnothing[5]=\left(n[3][0]-3^{*} n[1][2]\right)^{*}(n[3][0]+n[1][2])^{*} \\
& \left((n[3][0]+n[1][2]) *(n[3][0]+n[1][2])-3^{*}(n[2][1]+n[0][3])^{*}(n[2][1]+n[0][3])\right)+ \\
& \left(3^{*} n[2][1]-n[0][3]\right)^{*}(n[2][1]+n[0][3])^{*} \\
& \left(3^{*}(n[3][0]+n[1][2]) *(n[3][0]+n[1][2])\left(3^{*} n[2][1]+n[0][3]\right) *\left(3^{*} n[2][1]+n[0][3]\right)\right) ; \\
& \varnothing[6]=(n[2][0]-n[0][2])^{*}\left((n[3][0]+n[1][2])^{*}(n[3][0]+n[1][2])-(n[2][1]+n[0][3])^{*}\right. \\
& (n[2][1]+n[0][3]))+4^{*} n[1][1]^{*}(n[3][0]+n[1][2])^{*}(n[2][1]+n[0][3]) ; \\
& \varnothing[7]=\left(3^{*} \eta[2][1]-[0][3]\right)^{*}(n[3][0]+n[1][2])^{*}(n[3][0]+n[1][2])^{*}(\eta[3][0]+n[1][2])- \\
& \left.3^{*}(n[2][1]+n[0][3])^{*}(n[2][1]+n[0][3])\right)+\left(3^{*} n[1][2]-\right. \\
& \eta[3][0])^{*}(n[2][1]+n[0][3])^{*}\left(3^{*}(n[3][0]+n[1][2])^{*}(n[3][0]+n[1][2])-\right. \\
& \left.\eta[2][1]+n[0][3])^{\star}(n[2][1]+\eta[0][3])\right) \text {; }
\end{aligned}
$$




\subsection{Compare Reference and Sample Image}

In the map matching with invariant moments, the similarity between two images is determined by the correlation of the seven set of invariant moments of the images with those computed at each of the test locations. Database for reference and sample images are created. It is an array that contains seven set of invariant moment's [5].

Reference and sample images are compared by the given expression.

$$
R(u, v)=\sum_{i=1}^{7} \phi_{1 i} \phi_{2 i}(u, v) / \sqrt{\sum_{i=1}^{7} \phi_{1 i}{ }^{2} \sum_{i=1}^{7} \phi_{2 i}{ }^{2}(u, v)}
$$

Where

$\mathrm{R}(\mathrm{u}, \mathrm{v}) \rightarrow$ is the moment correlation at the test location $(\mathrm{u}, \mathrm{v})$.

$\phi_{1 i} \rightarrow$ the $\mathrm{i}^{\text {th }}$ invariant moment of the reference image.

$\phi_{2 i} \rightarrow$ the $\mathrm{i}^{\text {th }}$ invariant moment of the image.

In this application two tasks are created. In task 0 seven ( 0 to 7$)$ set of invariant moments for the reference image are sent to queue 0 . In task 1 seven ( 0 to 7$)$ set of invariant moments for the sample image are sent to queue 1 . This is shown in the Figure 2.
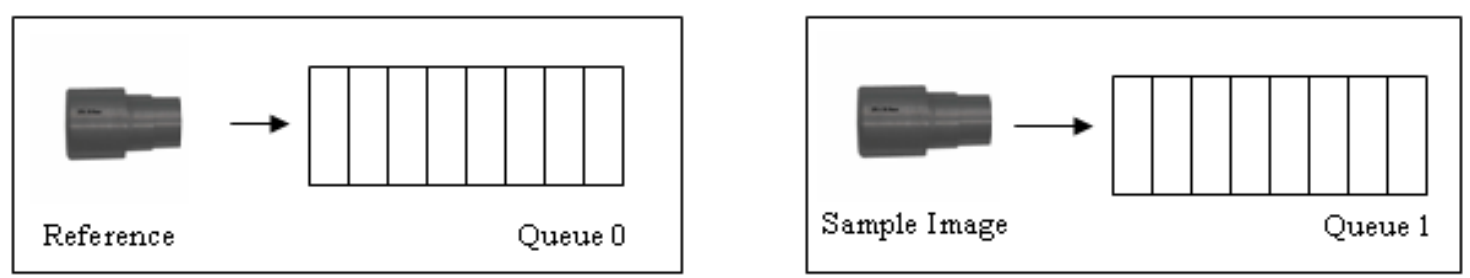

Figure 2: Sending Reference Image Moments into Queue 0 and Queue 1.

In this task 1 again the moments are received from the respective queues for image comparison [6]. This is explained in the Figure 3. 


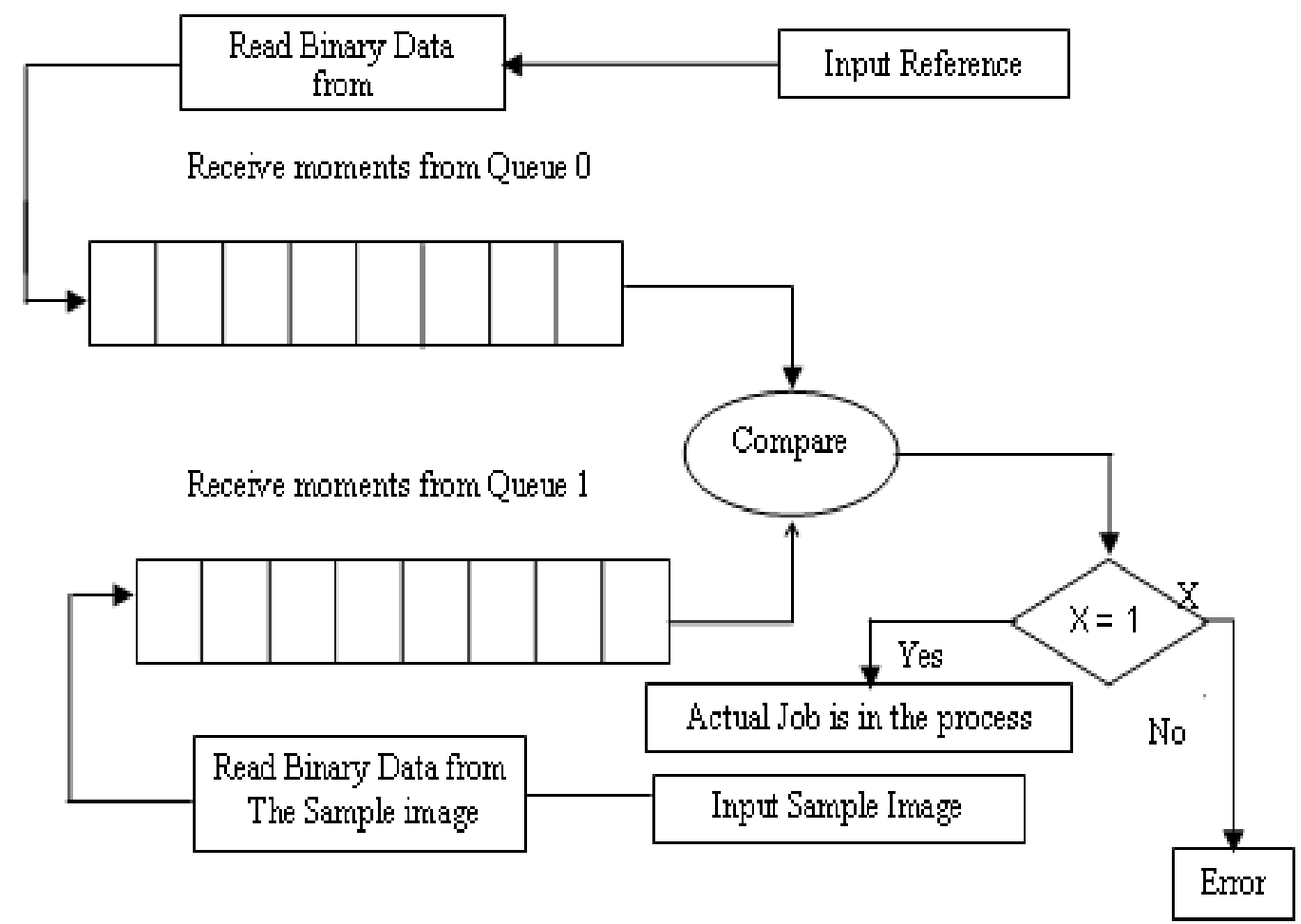

Figure 3: Flow Chart for Comparison of Sample and Reference Image.

Moments from queue 0 and moments from queue 1 are compared, if the value of the comparison is equal to one or very close to one then the actual job is in process else the actual job is not in process. Pictorial representation of robotics in online inspection and quality control using arm processor, for executing the application is shown in Figure 4.

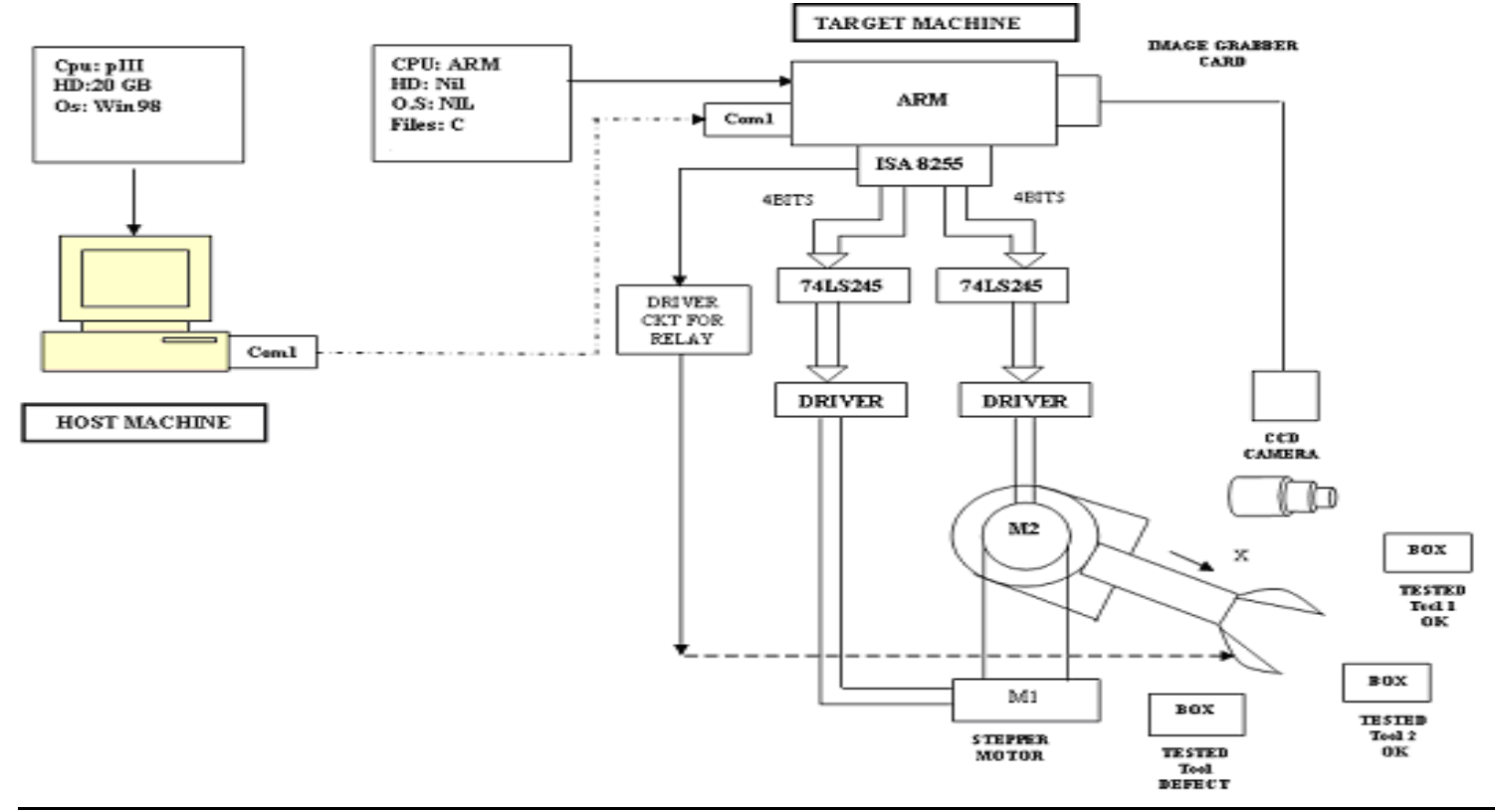

Figure 4: Pictorial Representation of On-Line Production Job Inspection Using Arm Processor. 
Dev Anand, Selveraj, Kumanan \& Ajith Bosco Raj: Robotics in Online Inspection and Quality Control...

\section{RESULTS OF INVARIANT MOMENTS FOR VARIOUS IMAGES}

The following images were tested using moment algorithm and the results are tabulated in Table I.

Table I: Seven set of Invariant Moments for Various Images.

\begin{tabular}{|c|c|c|}
\hline $\begin{array}{c}\text { Sl. } \\
\text { No }\end{array}$ & Image & Moments \\
\hline \multirow[t]{7}{*}{ INO } & & $\varnothing 1=0.0008690$ \\
\hline & & $\varnothing 2=0.0000000$ \\
\hline & & $\varnothing 3=0.0000000$ \\
\hline & & $\varnothing 4=0.0000000$ \\
\hline & & $\varnothing 5=0.0000000$ \\
\hline & & $\varnothing 6=0.0000000$ \\
\hline & & $\varnothing 7=0.0000000$ \\
\hline \multirow{7}{*}{2} & & $\varnothing 1=0.0001180$ \\
\hline & & $\varnothing 2=0.0000000$ \\
\hline & & $\varnothing 3=0.0000000$ \\
\hline & & $\varnothing 4=0.0000000$ \\
\hline & & $\varnothing 5=0.0000000$ \\
\hline & & $\varnothing 6=0.0000000$ \\
\hline & & $\varnothing 7=0.0000000$ \\
\hline \multirow{7}{*}{3} & & $\varnothing 1=0.0012050$ \\
\hline & & $\varnothing 2=0.0000010$ \\
\hline & & $\varnothing 3=0.0000000$ \\
\hline & & $\varnothing 4=0.0000000$ \\
\hline & & $\varnothing 5=0.0000000$ \\
\hline & & $\varnothing 6=0.0000000$ \\
\hline & & $\varnothing 7=0.0000000$ \\
\hline \multirow{7}{*}{4} & & $\varnothing 1=0.0010130$ \\
\hline & & $\varnothing 2=0.0000000$ \\
\hline & & $\varnothing 3=0.0000000$ \\
\hline & & $\varnothing 4=0.0000000$ \\
\hline & & $\varnothing 5=0.0000000$ \\
\hline & & $\varnothing 6=0.0000000$ \\
\hline & & $\varnothing 7=0.0000000$ \\
\hline \multirow{7}{*}{5} & & $\varnothing 1=0.0009550$ \\
\hline & & $\varnothing 2=0.0000000$ \\
\hline & & $\varnothing 3=0.0000000$ \\
\hline & & $\varnothing 4=0.0000000$ \\
\hline & & $\varnothing 5=0.0000000$ \\
\hline & & $\varnothing 6=0.0000000$ \\
\hline & & $\varnothing 7=0.0000000$ \\
\hline \multirow{7}{*}{6} & & $\varnothing 1=0.0010560$ \\
\hline & & $\varnothing 2=0.0000000$ \\
\hline & & $\varnothing 3=0.0000000$ \\
\hline & & $\varnothing 4=0.0000000$ \\
\hline & & $\varnothing 5=0.0000000$ \\
\hline & & $\varnothing 6=0.0000000$ \\
\hline & & $\varnothing 7=0.0000000$ \\
\hline
\end{tabular}


Six images are taken for image comparison. Image 1 is considered as a reference image and the remaining images are sample images. The images 2 and 3 are differing from the reference image. The remaining images are same but tilted by an angle. When comparing these images, if the reference and sample images are same, we will get the resultant value as one. If the sample image is same as reference image but tilted by an angle, the resultant value will be very close to one. If the Reference Image (RI) and Sample Images (SI) are different, the resultant value will be less than one. The results

Table II : Comparison between reference and sample images.

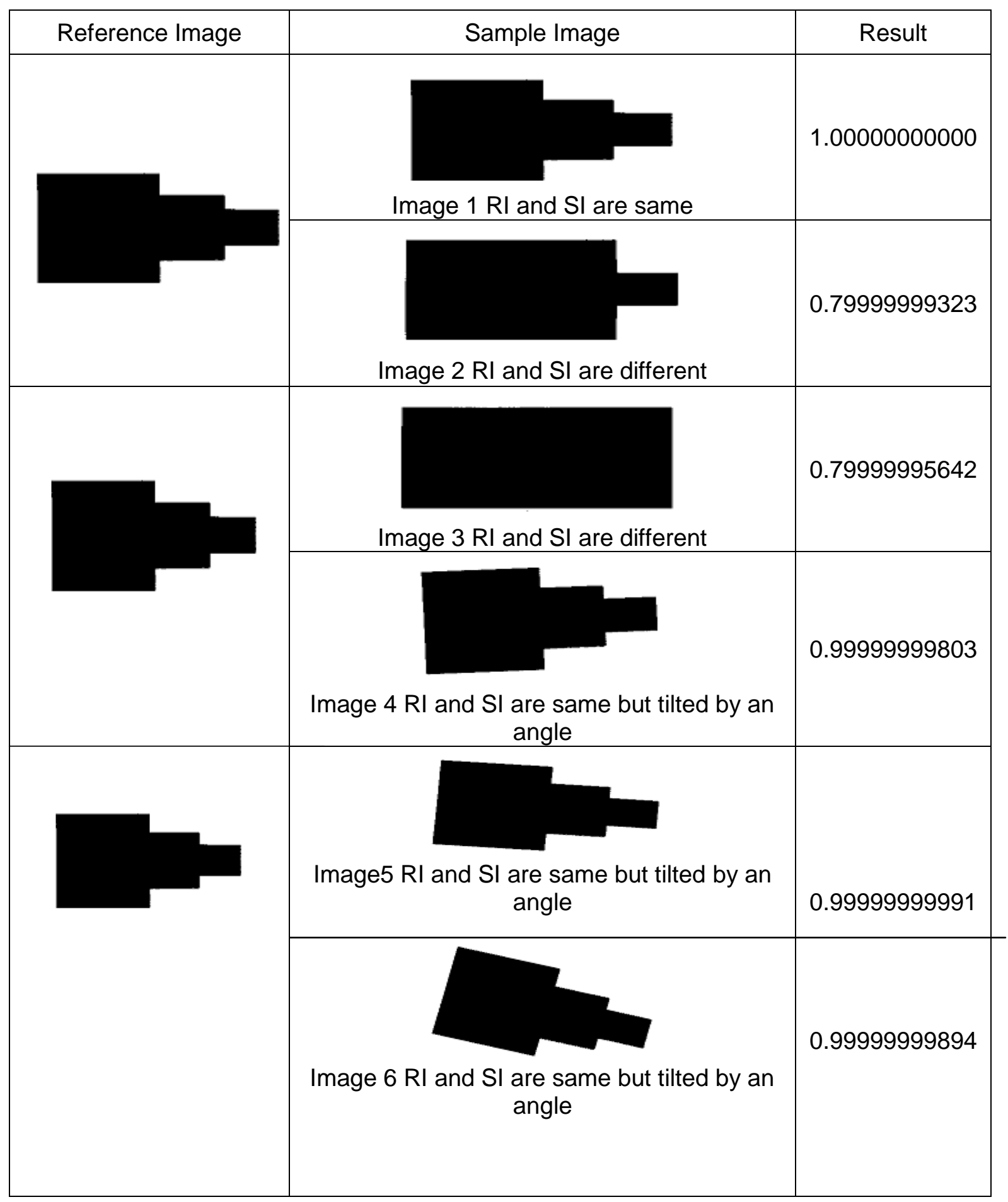




\section{CONCLUSION}

Automated system for inspection purposes using industrial robots have a significant potential. The development of more advanced, accurate and low cost sensors in combination with the flexibility of robots may provide solutions to many different tasks. The need for automated systems will also increase as the sensory capability of the human operator is removed. The objects presented to the robot are uniform and that the programming has been properly carried out, the resulting quality has generally a higher average than when human workers perform the operation. A definitely improved image results over the other shape analysis method and computer with ordinary operating system in terms of determinacy, scalability and redundancy. Using moment algorithm the ' $\mathrm{C}$ ' program had been developed and implemented.

\section{REFERENCES}

[1] Day, C.P. (1987): "Robot accuracy issues and methods of improvements", SME Robotics Today, 4 (1), 77-111

[2] Dev Anand, M.: Selvaraj, T.: Kumanan, S.: and Ajith Bosco Raj, T. (2006): "Robotics in online inspection and quality control", INCRUIS, 1-2, 317-323

[3] Ballard, D.H. (1982): “Computer Vision”, Prentice Hall India

[4] Maragos, P. (1987): "Tutorial on advances in morphological image processing and analysis", Optical Engineering, 26 (7)

[5] Iraklis, M. (1998): "Real-time computation of two-dimensional moments on binary images using image block representation", IEEE Trans. on Image Processing, 7 (11)

[6] Jan Flusser. (2000): "Redefined moment calculation using image block representation", IEEE Transactions on Image Processing, 9 (11) 